\title{
Improvement in the regulation of the vitamin $K$ antagonist acenocoumarol after a standard initial dose regimen: prospective validation of a prescription model
}

\author{
Johanna H. H. Van Geest-Daalderop • \\ Barbara A. Hutten · Nathalie C. V. Péquériaux · \\ Marcel Levi · Augueste Sturk
}

Published online: 13 February 2008

(C) The Author(s) 2008

\begin{abstract}
Background In a retrospective study we have developed a model which determines the dose of acenocoumarol based on the age of the patient and on the first INR obtained after a standard initial loading dose. The group of patients of this study was used as the control group of the present study. Aim The aim of this study was to prospectively validate the model and to assess whether the use of this model improves the quality of the treatment in the 0-2 months study period. Patients and methods In 197 patients the model was evaluated by (1) in the initial phase: comparison of INRs with the control group, after assessing the dose according to the model, and (2) in the $0-2$ months period: calculation of the percentage of time spent in the therapeutic target range compared to the control group. Furthermore, the eventual dose was compared to the dose of the model when the INRs were within the therapeutic target range for the first time and on two successive occasions. Results (1) When dosed according to the model, $50 \%$ of INRs in the total group were within the
\end{abstract}

J. H. H. Van Geest-Daalderop ( $\square)$ · N. C. V. Péquériaux Thrombosis Service, Department of Laboratory of Clinical Chemistry and Haematology, Jeroen Bosch Hospital, Helftheuvelweg 11, 's-Hertogenbosch 5222 AV,

The Netherlands

e-mail: j.vangeest@planet.nl

B. A. Hutten

Department of Clinical Epidemiology and Biostatistics,

Academic Medical Center, Amsterdam, The Netherlands

M. Levi

Department of Internal Medicine, Academic Medical Center, Amsterdam, The Netherlands

A. Sturk

Department of Clinical Chemistry, Academic Medical Center, Amsterdam, The Netherlands therapeutic target range compared to $45 \%$ in the control group, and (2) the percentage time spent within this range was 68 in the total group compared to 63 in the control group $(P=0.0013)$. When the INRs were within the range for the first time and successively twice, the eventual doses were similar to the model in 59 and $50 \%$, respectively. About $20 \%$ of the patients did not achieve two successive INRs within the range. Conclusions Using the model the quality of treatment improved. We advice to use a standardized individualized dose regimen at the initiation of vitamin $\mathrm{K}$ antagonist treatment.

Keywords Acenocoumarol - Initial dose regimen . Dose model $\cdot$ Eventual dose

\section{Introduction}

Adequate management of oral anticoagulant treatment with a vitamin $\mathrm{K}$ antagonist (VKA) is aimed at achieving the prothrombin time/international normalized ratio (PT/INR) in the therapeutic target range as soon as possible after the start of the treatment, and at subsequently maintaining the $\mathrm{PT} / \mathrm{INR}$ values in this range [1].

However, the individual correct dose, that may vary from patient-to-patient, is often found by trial and error in the first weeks after the initiation of the VKA treatment, sometimes resulting in fluctuating INR values and an adequate intensity of anticoagulation in a relatively low proportion of patients only. In the Netherlands, VKA treatment in the outpatient setting is carried out by specialized anticoagulation clinics. Cross-sectional studies in about 215,000 patients of these anticoagulation clinics, treated with acenocoumarol and phenprocoumon, showed that in the last few years the percentage of INR values 
within the therapeutic target range in the first 2 months after the start of the treatment was 62 (median), compared to $74 \%$ in the 3-6 months period after initiation and $78 \%$ after 6 months, respectively [2]. Another study [3] found that in the first 6 weeks after the start of acenocoumarol the INR values were only in $32.6 \%$ of patients and in only $32.4 \%$ of the time within the therapeutic target range [4].

In a previously reported retrospective study in outpatients with atrial fibrillation who started with acenocoumarol, we found a clear predictive correlation between the first INR (after an initial standard loading dose of 6 and $4 \mathrm{mg}$ acenocoumarol on day 1 and 2 , or $6 \mathrm{mg}, 4 \mathrm{mg}$ and $2 \mathrm{mg}$ on day 1,2 and 3, respectively) and the age of the patient on the eventual dose of the VKA [5]. Based on that correlation we developed a model to predict the dose of acenocoumarol after the initial loading dose.

In the present study we prospectively evaluated our model in outpatients with acenocoumarol.

\section{Patients and methods}

\section{Patients}

We prospectively evaluated our dose model of the initial dosing of the VKA acenocoumarol in outpatients managed by an anticoagulation clinic. The dose model was developed in our previously published retrospective study [5]. Both studies were carried out in the same anticoagulation clinic. We compared the results of the two studies, using the patients of the previous study as control group. In the previous study the dose was assessed based on the experience of the physician and in the present study according to the dose model. In both studies we studied patients with atrial fibrillation, and in the present study also patients with venous thromboembolism, as both indications for VKA treatment have the same therapeutic target range. In the present study data of patients with atrial fibrillation and of the total group (atrial fibrillation and venous thromboembolism) are shown. All eligible patients were included consecutively. We included 197 patients, 111 with atrial fibrillation and 86 with venous thromboembolism, who started VKA treatment with an initial loading dose of $6 \mathrm{mg}$ on day 1 and $4 \mathrm{mg}$ on day 2, or $6 \mathrm{mg}, 4 \mathrm{mg}$ and $2 \mathrm{mg}$ on day 1,2 and 3 , respectively, and who were planned to continue the anticoagulant treatment for at least 2 months. The starting doses were determined by the physicians who refer the patient to the anticoagulation clinic and these physicians were asked to start with the doses as mentioned above. Patients were included after confirmation by telephone of the correct intake of the initial loading dose. Patients took their medication at 6 p.m. and blood was collected in the morning. The target INR range was 2.0 3.5. All patients with venous thromboembolism also received therapeutic doses of low-molecular-weight heparin (LMWH) at least during the first 5 days and until two consecutive INRs were $\geq 2.0[6,7]$.

\section{Study procedure}

After the first 2 or 3 days of the intake of the initial loading dose acenocoumarol the INR was determined. The subsequent dose was based on the previously established formula, expressed in a model that takes into account the first INR value and the age of the patient and which is presented in Table 1 [5]. Taking into account the ranges of the confidence interval, we accepted a margin of $\pm 0.3 \mathrm{mg}$. To be able to assess the daily dose for each first INR and each decade of age, the physician used dose algorithms, derived from the mean dose of Table 1, dividing the tablet strengths as equally as possible. After at least 5 days of taking the dose according to the model, further doses were assessed, based on subsequent INR values, by the physician and by a computerized dosing program (ThromboseDienst AutomatiseringsSysteem (TDAS), Amsterdam, The Netherlands). This period of 5 days was chosen because after 5 days prothrombin that has the longest half-life $( \pm 60 \mathrm{~h})$ of the vitamin K-dependent coagulation factors, is likely to have reached a stable value. Patients were followed for 2 months after initiation of VKA treatment.

The data of the initial phase and the 2 months period were compared to the similar period in the previous study (control group). The quality of anticoagulation was assessed by two methods: (1) in the initial phase: the number of INR values within, below and above the therapeutic target range in patients of whom the dose was assessed according to the model in comparison with those of the control group, and (2) in the 0-2 months follow-up period: the percentages of time within, below and above the therapeutic target range as compared to the results of the control group.

Furthermore, to find the similarity between the eventual dose and the dose of the model, both doses were compared when the INRs were within the therapeutic target range for the first time and on two successive occasions (the maintenance dose).

We assessed the occurrence of thrombotic events and bleeding complications, by systematically asking patients at the time of blood sampling for signs and symptoms of these complications. All thrombotic events and major bleeding complications had to be confirmed by objective means. A bleeding was defined as major if it was fatal, intracranial, intra-articular, or leading to hospital admission or blood transfusion. All other bleedings were considered minor. 
Table 1 The prescribed dose based upon the age and the first INR value after taking an initial dose regimen of 6-4-(2) mg acenocoumarol [5]

\begin{tabular}{|c|c|c|c|c|c|c|c|c|c|c|c|c|}
\hline \multirow{2}{*}{$\begin{array}{l}\text { Age (yrs) } \\
\text { First INR }\end{array}$} & \multicolumn{2}{|l|}{$40-50$} & \multicolumn{2}{|l|}{$50-60$} & \multicolumn{2}{|l|}{$60-70$} & \multicolumn{2}{|l|}{$70-80$} & \multicolumn{2}{|l|}{$80-90$} & \multicolumn{2}{|c|}{$90-100$} \\
\hline & Mean & $95 \% \mathrm{CI}$ & Mean & $95 \% \mathrm{CI}$ & Mean & $95 \% \mathrm{CI}$ & Mean & $95 \% \mathrm{CI}$ & Mean & $95 \% \mathrm{CI}$ & Mean & $95 \% \mathrm{CI}$ \\
\hline 1 & 4.5 & $4.3-4.8$ & 4.4 & $4.2-4.6$ & 4.3 & $4.1-4.5$ & 4.2 & $4.0-4.4$ & 4.1 & $3.8-4.4$ & 4.0 & $3.7-4.3$ \\
\hline 1.5 & 3.9 & $3.7-4.1$ & 3.8 & $3.6-3.9$ & 3.7 & $3.5-3.8$ & 3.5 & $3.4-3.7$ & 3.4 & $3.2-3.6$ & 3.3 & $3.1-3.6$ \\
\hline 2 & 3.5 & $3.2-3.6$ & 3.3 & $3.2-3.4$ & 3.2 & $3.1-3.3$ & 3.1 & $2.9-3.2$ & 3.0 & $2.8-3.1$ & 2.9 & $2.6-3.1$ \\
\hline 2.5 & 3.0 & $2.8-3.2$ & 2.9 & $2.8-3.0$ & 2.8 & $2.7-2.9$ & 2.7 & $2.6-2.8$ & 2.6 & $2.4-2.7$ & 2.5 & $2.3-2.7$ \\
\hline 3 & 2.7 & $2.5-2.9$ & 2.6 & $2.5-2.7$ & 2.5 & $2.4-2.6$ & 2.4 & $2.3-2.5$ & 2.3 & $2.1-2.4$ & 2.2 & $2.0-2.4$ \\
\hline 3.5 & 2.5 & $2.3-2.7$ & 2.4 & $2.2-2.5$ & 2.3 & $2.2-2.3$ & 2.1 & $2.1-2.2$ & 2.0 & $1.9-2.2$ & 1.9 & $1.7-2.1$ \\
\hline 4 & 2.3 & $2.0-2.5$ & 2.1 & $2.0-2.3$ & 2.0 & $1.9-2.1$ & 1.9 & $1.8-2.0$ & 1.8 & $1.7-1.9$ & 1.7 & $1.5-1.9$ \\
\hline 4.5 & 2.1 & $1.8-2.3$ & 1.9 & $1.8-2.1$ & 1.8 & $1.7-1.9$ & 1.7 & $1.6-1.8$ & 1.6 & $1.5-1.8$ & 1.5 & $1.3-1.7$ \\
\hline 5 & 1.9 & $1.6-2.1$ & 1.8 & $1.6-1.9$ & 1.7 & $1.5-1.8$ & 1.6 & $1.4-1.7$ & 1.4 & $1.3-1.6$ & 1.3 & $1.1-1.5$ \\
\hline 5.5 & 1.7 & $1.5-2.0$ & 1.6 & $1.4-1.8$ & 1.5 & $1.4-1.6$ & 1.4 & $1.3-1.5$ & 1.3 & $1.1-1.4$ & 1.2 & $1.0-1.4$ \\
\hline 6 & 1.6 & $1.3-1.8$ & 1.5 & $1.3-1.7$ & 1.4 & $1.2-1.5$ & 1.3 & $1.1-1.4$ & 1.1 & $1.0-1.3$ & 1.0 & $0.8-1.2$ \\
\hline 6.5 & 1.4 & $1.2-1.7$ & 1.3 & $1.1-1.5$ & 1.2 & $1.1-1.4$ & 1.1 & $1.0-1.3$ & 1.0 & $0.8-1.2$ & 0.9 & $0.7-1.1$ \\
\hline 7 & 1.3 & $1.0-1.6$ & 1.2 & $1.0-1.4$ & 1.1 & $0.9-1.3$ & 1.0 & $0.8-1.2$ & 0.9 & $0.7-1.1$ & 0.8 & $0.6-1.0$ \\
\hline 7.5 & 1.2 & $0.9-1.5$ & 1.1 & $0.9-1.3$ & 1.0 & $0.8-1.2$ & 0.88 & $0.7-1.1$ & 0.8 & $0.6-1.0$ & 0.7 & $0.4-0.9$ \\
\hline 8 & 1.1 & $0.8-1.4$ & 1.0 & $0.8-1.2$ & 0.9 & $0.7-1.1$ & 0.77 & $0.6-1.0$ & 0.7 & $0.5-0.9$ & 0.6 & $0.3-0.8$ \\
\hline 8.5 & 1.0 & $0.7-1.3$ & 0.9 & $0.6-1.1$ & 0.8 & $0.6-1.0$ & 0.67 & $0.5-0.9$ & 0.6 & $0.4-0.8$ & 0.5 & $0.2-0.7$ \\
\hline 9 & 0.9 & $0.6-1.2$ & 0.8 & $0.5-1.1$ & 0.7 & $0.5-0.9$ & 0.58 & $0.4-0.8$ & 0.5 & $0.3-0.7$ & 0.4 & $0.1-0.6$ \\
\hline 9.5 & 0.8 & $0.5-1.1$ & 0.7 & $0.4-1.0$ & 0.6 & $0.4-0.8$ & 0.49 & $0.3-0.7$ & 0.4 & $0.2-0.6$ & 0.3 & $0.0-0.5$ \\
\hline 10 & 0.7 & $0.4-1.1$ & 0.6 & $0.4-0.9$ & 0.5 & $0.3-0.8$ & 0.41 & $0.2-0.6$ & 0.3 & $0.1-0.5$ & 0.2 & -0.1 to 0.4 \\
\hline
\end{tabular}

The first column on the left presents the first INRs and the other columns present the mean daily doses and $95 \%$ confidence intervals belonging to these first INRs and the various age groups. The dose was calculated according to the formula: mean daily dose $=5.03-1.65 * \ln$ (first INR) $-0.01 *$ age (years)

INR determination

Blood collection took place with Vacutainer (Becton Dickinson Vacutainer Systems, Beliver Industrial Estate, Plymouth, UK), containing a citrate solution of $0.109 \mathrm{~mol} / \mathrm{l}$. The PT/INR was determined immediately upon plasma preparation with a STA Rack (Roche Diagnostics GmbH, Mannheim, Germany) using Hepato Quick (Diagnostica Stago, Asnières, France) batches with an ISI of 0.9-0.96.

\section{Statistical analysis}

Continuous variables were compared using the two sample $t$-test. Chi-square tests were applied to compare distributions of dichotomous data. The quality of the VKA treatment was estimated by the percentage of time spent in the therapeutic target range (INR 2.0-3.5) and was calculated using linear interpolation [4]. This method assumes that INR values between two consecutive measurements vary linearly between the values of the first and the second measurements. Differences in percentage time spent below, within and above the therapeutic target range between the retrospective and prospective cohort were evaluated using linear regression analysis. By means of multivariate models adjustments were made stepwise (backward) for potential confounders, such as age, gender, startdose and duration of VKA treatment. All data were analysed using SAS 9.1 (SAS Institute, Cary, NC, USA).

\section{Results}

\section{Study population}

All 197 patients confirmed by telephone the correct intake of the starting dose of 6-4 or 6-4-2 mg acenocoumarol taken around 6 p.m. and all continued the VKA treatment during the 2 months of the study. Eleven of the patients with atrial fibrillation had medication (possibly) interfering with acenocoumarol already at the start of the treatment: methotrexate, levothyroxin, thiamazole, colchicine, allopurinol, cholestyramin and carbamazepine. One patient started with prednisone during the study period. Twelve patients with venous thromboembolism had (possibly) interfering medication, also from the start of the treatment: disulfiram, allopurinol, azathioprine, levothyroxin, prednisone, dexamethasone, fenytoine and carbamazepine. Three patients started medication during the study period: citalopram, allopurinol and colchicine. Ten patients stopped 
acenocoumarol for 1 or more days (maximum 3 days) for various reasons during the two months follow-up. In four patients vitamin $\mathrm{K}$ was administered for a high INR (in the absence of bleeding). Ten patients had a malignancy (six patients in the venous thromboembolism group). There were no thrombotic events and major bleedings did not occur. Seventeen patients had a minor bleeding.

Table 2 shows the clinical characteristics of the patients in the $0-2$ months follow-up period as compared to the patients of the control group. There were no statistically significant differences between the results of the groups starting with 6-4 or 6-4-2 (data not shown). In the present study the mean age was lower than in the control group (64 \pm 10 in the group with atrial fibrillation and $61 \pm 14$ years in the total group versus $69 \pm 10$ in the control group). Somewhat more patients in the present study had a first INR after the initial dose regimen below 2.0 (23\% in the group with atrial fibrillation and $25 \%$ in the total group versus $16 \%$ in the control group), less patients above 3.5 (12\% and $13 \%$ versus $35 \%$, respectively) and more patients had a first INR within the therapeutic target range (66\% and $62 \%$ versus $49 \%$, respectively). We may assume that this is caused by the differences in age of the patients, as mentioned above. This also results in a higher mean daily dose $(2.8 \pm 1.0$ versus $2.2 \pm 0.8)$.

Initial phase: INR values after assessment of the dose according to the model compared to the control group

The data of the patients in the present study were compared with those of the control group in the initial phase when the dose was taken for a minimum of 5 days. In the present study the dose was assessed according to the dose of the model and in the control group by an experienced physician. Table 3 shows the results. In the present study in $48 \%$ of the group with atrial fibrillation and in $50 \%$ of the total group the correct dose was assessed achieving INR values within the therapeutic target range, compared to $45 \%$ in the control group. The INR values were below the therapeutic target range to a lesser extent in the present study compared to the control group (13 and $16 \%$ versus $40 \%$ ) and to a larger extent above the range (39 and $34 \%$ versus $15 \%$ ). In the present study the data of the groups with atrial fibrillation and of the total group show a considerable similarity.

The $0-2$ months period: percentage time within the therapeutic target range in the present study compared to the control group

The quality of the VKA treatment in the 2 months study period is shown in Table 4. In the present study the percentage time spent within the therapeutic target range was 70 in patients with atrial fibrillation and 68 in the total group, compared to 63 in the control group. For both patients groups in the present study this difference was statistically significant $(P=0.0023$ and $P=0.0013$, respectively) after adjustment for the confounders age and duration of treatment. The increase of this percentage is mainly due to a decrease of the percentage of INRs $<2.0$. In the present study the data of the groups with atrial fibrillation and of the total group also are similar.

Table 2 Clinical characteristics of the patients on acenocoumarol with atrial fibrillation (AF) and venous thromboembolism (VTE) in the 0-2 months follow-up period

\begin{tabular}{|c|c|c|c|c|c|}
\hline & \multirow{3}{*}{$\begin{array}{l}\text { Control group } \\
\text { AF } \\
n=284\end{array}$} & \multicolumn{4}{|c|}{ Present study } \\
\hline & & \multicolumn{2}{|l|}{$\mathrm{AF}$} & \multicolumn{2}{|c|}{$\mathrm{AF}+\mathrm{VTE}$} \\
\hline & & $n=111$ & $P$-value* & $n=197$ & $P$-value* \\
\hline Age (years) & $69 \pm 10$ & $64 \pm 10$ & 0.0002 & $61 \pm 14$ & $<0.0001$ \\
\hline Male gender $(\%)$ & $184(65)$ & $78(70)$ & 0.3 & $108(55)$ & 0.03 \\
\hline \multicolumn{6}{|l|}{ Starting dose, $n(\%)$} \\
\hline $6-4$ & $144(51)$ & $38(34)$ & 0.0023 & $66(34)$ & 0.001 \\
\hline $6-4-2$ & $140(49)$ & $73(66)$ & & $131(66)$ & \\
\hline \multicolumn{6}{|l|}{ First INR, $n(\%)$} \\
\hline$<2.0$ & 45 (16) & $25(23)$ & $<0.0001$ & $50(25)$ & $<0.0001$ \\
\hline $2.0-3.5$ & $139(49)$ & $73(66)$ & & $122(62)$ & \\
\hline$>3.5$ & $100(35)$ & $13(12)$ & & $25(13)$ & \\
\hline Mean daily dosage (mg) & $2.2 \pm 0.8$ & $2.6 \pm 0.8$ & $<0.0001$ & $2.8 \pm 1.0$ & $<0.0001$ \\
\hline
\end{tabular}

* Control group versus present study

In the control group the data of patients with AF and in the present study the data of patients with AF and of AF + VTE are shown. The dose of patients in the control group was assessed by experienced physicians and in the present study according to the model 
Table 3 Initial phase: comparison of INR values after assessment of the dose of acenocoumarol according to the model in the present study with assessment by an experienced physician in the control group

\begin{tabular}{llll}
\hline & Control group & Present study & \\
\cline { 3 - 4 } & $\mathrm{AF}$ & $\mathrm{AF}$ & $\mathrm{AF}+\mathrm{VTE}$ \\
$n=284$ & $n=111$ & $n=197$ \\
\hline INRs, $n(\%)$ & & $14(13)$ & $31(16)$ \\
Below & $114(40)$ & $54(48)$ & $98(50)$ \\
Within & $128(45)$ & $43(39)$ & $68(34)$ \\
Above & $42(15)$ & & \\
\hline
\end{tabular}

In the control group the data of patients with atrial fibrillation (AF) and in the present study the data of patients with AF and with $\mathrm{AF}+$ venous thromboembolism (VTE) are shown. The number of INRs below, within and above the therapeutic target range are presented for patients of the control group and in the present study who started with an initial loading dose of 6-4-(2) mg acenocoumarol and who took the dose algorithm for a minimum of 5 days

Table 4 The 0-2 months period: quality of VKA treatment of the patients on acenocoumarol of the control group and in the present study

\begin{tabular}{|c|c|c|c|c|c|}
\hline & \multirow{3}{*}{$\begin{array}{l}\text { Control group } \\
\text { AF } \\
n=284\end{array}$} & \multicolumn{4}{|c|}{ Present study } \\
\hline & & \multicolumn{2}{|l|}{$\mathrm{AF}$} & \multicolumn{2}{|c|}{$\mathrm{AF}+\mathrm{VTE}$} \\
\hline & & $n=111$ & $P$-value* & $n=197$ & $P$-value* \\
\hline \multicolumn{6}{|c|}{$\%$ time spent in INR ranges (SD) } \\
\hline$<2.0$ & $22(26)$ & $11(20)$ & $<0.0001$ & $10(17)$ & $<0.0001 \#$ \\
\hline $2.0-3.5$ & $63(27)$ & $70(28)$ & $0.0023 \#$ & $68(28)$ & $0.0013 \#$ \\
\hline$>3.5$ & $14(22)$ & $19(26)$ & 0.0824 & $22(28)$ & $0.0088 \nRightarrow$ \\
\hline
\end{tabular}

* Control group versus present study

\# $P$-values are adjusted for the variables age and duration of treatment which remained in the model after stepwise backward regression analyses

In the control group the data of patients with atrial fibrillation (AF) and in the present study the data of patients with $\mathrm{AF}$ and with $\mathrm{AF}+$ venous thromboembolism (VTE) are shown. The dose of the patients in the control group was assessed by experienced physicians and in the present study according to the model. The percentages time spent below, within or above the therapeutic target range are shown in the $0-2$ months follow-up period

Comparison of eventual doses with doses of the model, when the INRs were in the therapeutic target range for the first time after the start and successively twice

To be able to compare the eventual doses to the doses of the model in the present study, an analysis was performed on the data obtained in patients with the INR values for the first time in the therapeutic target range and successively twice (maintenance dose) in the 2 months study period after taking a consistent dose algorithm for a minimum of 5 days.
When the INR value was in the therapeutic target range for the first time, in $116(59 \%)$ of the total group of 197 patients the eventual dose was similar to the dose of the model, in $49(25 \%)$ patients the eventual dose was lower and in 27 (14\%) patients the dose was higher than the dose of the model. In five $(2 \%)$ patients the INR values did not achieve the therapeutic target range at all in the study period.

When the INR values were in the therapeutic range successively twice (maintenance dose), 99 (50\%) patients of the total group had an eventual dose similar to the dose of the model, in $33(17 \%)$ patients the eventual dose was lower and in $25(13 \%)$ patients higher than the dose of the model, and, remarkably, in 40 patients (20\%) the INR values fluctuated and did not achieve the therapeutic target range consecutively twice in the study period. These fluctuating INR values were found to the same extent in patients with atrial fibrillation and venous thromboembolism (21 and 19 patients, respectively). Of the remaining 157 patients who achieved the maintenance dose in the study period, the differences between the eventual doses and the doses of the model across the patient population are presented in Fig. 1. In this group of 157 patients, the percentage of patients with an eventual dose similar to the dose of the model was 63 . The eventual doses that were different from the doses of the model were found in all ages and were not connected with high or low first INR values after the initial loading dose. The mean number of INR determinations in the 40 patients not achieving the maintenance dose in the 0-2 months study period was higher than in the 157 patients with two consecutive INRs values within the therapeutic target range, 6.3 (SD 1.6) and 5.2 (SD 1.3), respectively.

In general it is difficult to predict in daily practice if the eventual dose will differ from the dose of the model without further laboratory investigation. In our study only a small number of patients had medication inhibiting or potentiating the effect of VKAs, or concomitant diseases as malignancy.

\section{Discussion}

In this study we prospectively evaluated a newly developed model that is able to predict the correct dose of acenocoumarol after an initial loading dose of 6-4(-2) mg, based on the first INR value after the loading regimen and the age of the patient [5]. In the outpatient setting it is not very practical to carry out an INR determination several times a week, so the use of this model may help to avoid a longlasting period with fluctuating INR values before the correct dose has been established.

The extent of decrease of the coagulation factors according to their half-lives-in the initial phase 


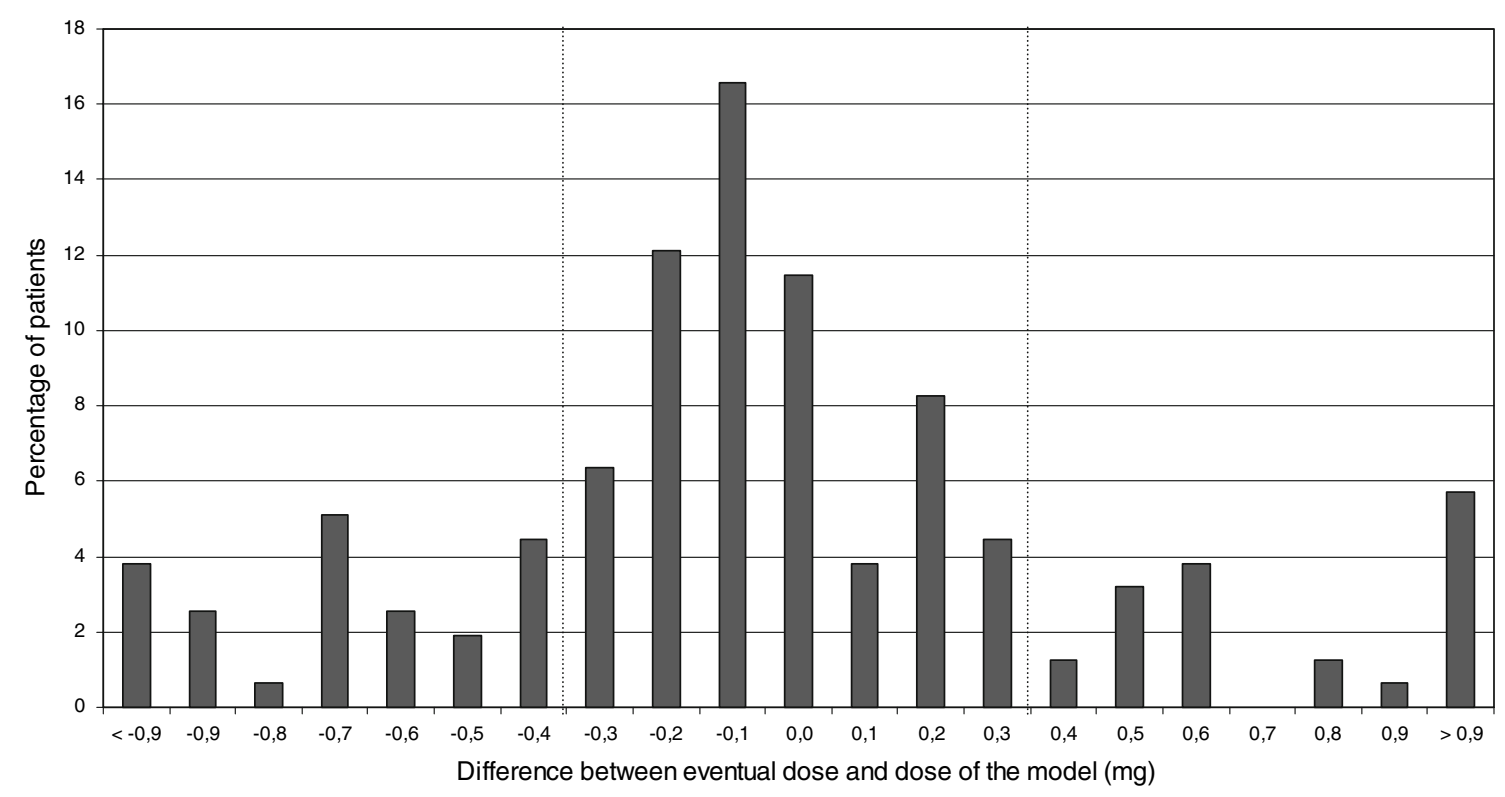

Fig. 1 The percentages of patients with differences between the eventual dose and the dose of the model in the $0-2$ months period. The data of 157 patients are presented. The eventual doses were compared to the doses of the model when the INR values were within

particularly factor VII with his short half-life of $5 \mathrm{~h}-$ is important for the extent of increase of the INR [8]. Since this decrease varies between patients, the question may be posed if the first INR after a standard initial dose regimen is a suitable predictor for the dose. However, in a considerable number of patients a clear relationship was found between the first INR, the dose of the model and the eventual dose, so it is an appropriate parameter to use.

Patients were included consecutively and not by randomization, because all physicians of the anticoagulation clinic knew our published findings of the retrospective study and this might influence the results because it is likely that those findings had affected the way they assessed the appropriate dose of the vitamin $\mathrm{K}$ antagonists.

The mean age of the patients in the control group was higher than in the present study. We assume that this difference did not influence the results of our study since an appropriate dose could be assessed for each decade of age and each first INR after the initial loading dose. We can only guess about the cause of this difference in age. Possibly, in the previous study more elderly patients were included, because this study was carried in the late nineties, after the publication of the large studies that showed the benefit of VKA treatment also in elderly patients with atrial fibrillation, which may have caused an extra wave of patients starting anticoagulant treatment.

In the present study the use of the dose according to the model during the 2 months follow-up period resulted in a percentage of time spent within the therapeutic target range of $70 \%$ in the group with atrial fibrillation and $68 \%$ in the the therapeutic range successively twice after the initial dose regimen of 6-4-(2) $\mathrm{mg}$ acenocoumarol and after taking a consistent dose algorithm for a minimum of 5 days. Between the dotted lines the eventual doses are similar to the mean doses of the model

total group (Table 4). The physicians who assessed the doses in the control group had a really great experience resulting in a relatively high percentage within the therapeutic target range. Nevertheless, compared to this control group (63\% of time spent within the range) and a previously performed national cross-sectional study in about 215,000 patients $(62 \%$ of INRs within the range) [2, 9], these results show a significant and clinically relevant improvement. We feel it is an important result of our study that the use of this model to assess the dose after a standard initial regimen is preferable to the assessment of the dose by trial and error, even though the model dose is correct for about $50 \%$ of the patients. It may seem rather disappointing that it has proven to be impossible to develop a model applicable for a larger part of the patients. In a substantial part of the patients the dose according to the model was not correct and the eventual doses varied from the doses of the model quite widely (Fig. 1). Nevertheless, the model proved to be a useful tool to more precisely define the optimal dose of VKA in the initial period. Using the model, a relatively high percentage of INRs within the therapeutic target range will be achieved consistently, the assessment of the first dose after the starting dose is independent of the experience of the physician, and a good quality of treatment is provided in the first weeks after the start. We propose that the model can be used as a starting point for all patients, taking into account that an adjustment of the dose may be necessary in a substantial number of patients.

The improved result was mainly due to a decrease of the percentage below the therapeutic target range. Based on 
their experience physicians are able to prescribe the proper dose in quite a lot of patients (Table 3). However, for the other patients they tend to prescribe lower doses out of fear for bleeding complications, and apparently they need a longer period of trial and error to find the correct dose, resulting in a lower percentage of time within the therapeutic target range. Also for warfarin, using a dose model-and also a computerized system-appears to improve the quality considerably [10]. In patients with atrial fibrillation and venous thromboembolism the lower limit of the INR value to be achieved and maintained is 2.0 $[11,12]$, and for that reason a rapid achievement of an INR $>2.0$ after the start of the treatment is important. For venous thromboembolism the period of administration of one of the LMWHs bridging the initial period of VKA treatment may be reduced, which further emphasizes the benefit of finding rapidly the proper dose.

Strikingly, a side result of this study is that $20 \%$ of the patients, both with atrial fibrillation and venous thromboembolism, did not achieve a stable maintenance dose in the 0-2 months period. For these patients with fluctuating INR values it may not be possible to develop a dose model. The effect of the VKA treatment is influenced by many factors. Besides the compliance of the patient, concomitant diseases, interaction of other medicines and vitamin $\mathrm{K}$ in the food influence the INR value [1]. A poor dietary intake of vitamin $\mathrm{K}$ may cause less stability and a low and steady supplementation of vitamin $\mathrm{K}$ to patients using VKAs antagonists may prevent an instable treatment $[13,14]$. The short half-life of acenocoumarol in combination with the short half-life of factor VII [15], and the intake of a dose algorithm with uneven daily doses of acenocoumarol [16] also may have an effect on the INR. Moreover, recently the genotypes of both the systems CYP2C9 and VKORC1 have been found to influence the stability and dose of VKAs [17-19]. Polymorphisms of one genotype or of a combination of both genotypes show a higher risk of excessive anticoagulation, a decreased chance to achieve stability and a lower dose requirement. It would be interesting to compare the genotypes of CYP2C9 and VKORC1 of the patients of our present study in a new study. Possibly, in the future it may prove useful to determine these genotypes of the patient to determine the dose requirements of VKAs [19-21]. Moreover, an individual time within the therapeutic target range of $<45 \%$ in the first 30 days of treatment predict a poor quality during the total period of VKA treatment and a higher risk of thromboembolic events and major bleedings [22].

Whether a loading dose of warfarin is to be recommended or not, is still a matter of debate $[1,23]$. In the present study the mean daily dose was $2.8 \mathrm{mg}$ acenocoumarol. We propose that an initial dose of $6-4-2 \mathrm{mg}$ acenocoumarol, exceeding this mean daily dose to a small extent, is appropriate for patients younger than 70-75 years [5]. An initial dose regimen of e.g. 4-2-1 mg may be prescribed to the elderly. A model to assess the dose for this group of patients can be developed according to the same method carried out in our previous study [5]. To be able to develop models for the assessment of the dose after the start of VKA treatment, based on age and the first INR, agreements about the initial dose regimens have to be made first.

In conclusion, our study shows that the use of a model increases the percentage time spent in the therapeutic target range in the 0-2 months period, compared to finding the dose by trial and error. Though the model is not correct for all patients, the use of the model is advisable because it provides guidelines in all patients which may subsequently lead to adjusted dosages depending on the individual patient.

Open Access This article is distributed under the terms of the Creative Commons Attribution Noncommercial License which permits any noncommercial use, distribution, and reproduction in any medium, provided the original author(s) and source are credited.

\section{References}

1. Ansell J, Hirsh J, Poller L et al (2004) The pharmacology and management of the vitamin K antagonists: the seventh ACCP conference on antithrombotic and thrombolytic therapy. Chest 126:204S-223S

2. Van Geest-Daalderop JHH, Sturk A, Levi M et al (2004) Extent and quality of anticoagulation treatment with coumarin derivatives by the Dutch thrombosis services. Ned Tijdschr Geneesk 148:730-735. Dutch

3. Gadisseur APA, van der Meer FJM, Adriaansen HJ et al (2002) Therapeutic quality control of oral anticoagulant therapy comparing the short-acting acenocoumarol and the long-acting phenprocoumon. Br J Haematol 117:940-946

4. Rosendaal FR, Cannegieter SC, van der Meer FJM, Briët E (1993) A method to determine the optimal intensity of oral anticoagulation therapy. Thromb Haemost 39:236-239

5. Van Geest-Daalderop JHH, Hutten BA, Sturk A, Levi M (2003) Age and first INR after initiation of oral anticoagulant therapy with acenocoumarol predict the maintenance dosage. J Thromb Thrombolysis 15:197-203

6. Büller HR, Agnelli G, Hull RD et al (2004) Antithrombotic therapy for venous thromboembolic disease: the seventh ACCP conference on antithrombotic and thrombolytic therapy. Chest 126:401S-428S

7. Koopman MMW, Prandoni P, Piovella $F$ et al, The Tasman Study Group (1996) Treatment of venous thrombosis with intravenous unfractionated heparin administered in the hospital as compared with subcutaneous low-molecular-weight heparin administered at home. N Engl J Med 334:682-687

8. Weiss P, Soff GA, Halkin H, Seligsohn U (1987) Decline of proteins $\mathrm{C}$ and $\mathrm{S}$ and factors II, VII, IX and X during the initiation of warfarin therapy. Thromb Res 45:783-790

9. Hutten BA, Prins MH, Redekop WK et al (1999) Comparison of three methods to assess therapeutic quality control of treatment with vitamin $\mathrm{K}$ antagonists. Thromb Haemost 82:1260-1263

10. Crowther MA (2003) Oral anticoagulant initiation: rationale for the use of warfarin dosing nomograms. Semin Vasc Med 3:255260 
11. Hylek EM, Go AS, Chang Y et al (2003) Effect of intensity of oral anticoagulation on stroke severity and mortality in atrial fibrillation. N Engl J Med 349:1019-1026

12. Kearon C, Ginsberg JS, Kovacs MJ, et al, Extended low-intensity anticoagulation for thromboembolism investigators (2003) Comparison of low-intensity warfarin therapy with conventionalintensity warfarin therapy for long-term prevention of recurrent venous thromboembolism. N Engl J Med 349:631-639

13. Rombouts EK, Rosendaal FR, van der Meer FJM (2007) Daily vitamin $\mathrm{K}$ supplementation improves anticoagulant stability. J Thromb Haemost 5:2043-2048

14. Sconce E, Avery P, Wynne H, Kamali F (2007) Vitamin K supplementation can improve stability of anticoagulation for patients with unexplained variability in response to warfarin. Blood 109:2419-2423

15. Van Geest-Daalderop JHH, Hutten BA, Péquériaux NCV et al (2007) The influence on INRs and coagulation factors of the time span between blood sample collection and intake of phenprocoumon or acenocoumarol: consequences for the assessment of the dose. Thromb Haemost 98:738-746

16. Marco F, Gutiérrez JI, Orkolaga K et al (2006) Patients who take uneven doses of acenocoumarol exhibit significant fluctuating levels of anticoagulation. Blood Coagul Fibrinolysis 17:509-512

17. Bodin L, Verstuyft C, Tregouet DA et al (2005) Cytochrome P450 2C9 (CYP2C9) and vitamin $\mathrm{K}$ epoxide reductase (VKORC1) genotypes as determinants of acenocoumarol sensitivity. Blood 106:135-140

18. Schalekamp T, Brassé BP, Roijers JFM et al (2006) VKORC1 and CYP2C9 genotypes and acenocoumarol anticoagulation status: interaction between both genotypes affects overanticoagulation. Clin Pharmacol Ther 80:13-22

19. Oldenburg J, Bevans CG, Fregin A, Geisen C, Müller-Reible C, Watzka M (2007) Current pharmacogenetic developments in oral anticoagulation therapy: the influence of variant VKORC1 and CYP2C9 alleles. Thromb Haemost 98:570-578

20. Sconce EA, Kahn TI, Wynne HA, Avery P, Monkhouse L, King BP, Wood P, Kesteven P, Daly AK, Kamali F (2005) The impact of CYP2C9 and VKORC1 genetic polymorphism and patient characteristics upon warfarin dose requirements: proposal for a new dosing regimen. Blood 106:2329-2333

21. Carlquist JF, Horne BD, Muhlestein JB, Lappé DL, Whiting BM, Kolek MJ, Clarke JL, James BC, Anderson JL (2006) Genotypes of the cytochrome p450 isoform, CYP2C9, and the vitamin K epoxide reductase complex subunit 1 conjointly determine stable warfarin dose: a prospective study. J Thromb Thrombolysis 22:191-197

22. Veeger NJGM, Piersma-Wichers M, Tijssen JGP et al (2005) Individual time within target range in patients treated with vitamin $\mathrm{K}$ antagonists: main determinant of quality of anticoagulation and predictor of clinical outcome. A retrospective study of 2300 consecutive patients with venous thromboembolism. Br J Haematol 128:513-519

23. Kovacs MJ, Rodger M, Anderson DR et al (2003) Comparison of $10-\mathrm{mg}$ and $5-\mathrm{mg}$ warfarin initiation nomograms together with low-molecular-weight heparin for outpatient treatment of acute venous thromboembolism. A randomized, double-blind, controlled trial. Ann Intern Med 138:714-719 